\title{
Clinical Features of Diffuse Leptomeningeal Glioneuronal Tumor With the First Symptom of Headache and Decreased Vision Misdiagnosed as Venous Sinus Thrombosis
}

\author{
Yi Bao a, b, Yajie $\mathrm{Hu}^{\mathrm{a}}$, Yayong Ding ${ }^{\mathrm{a}}$, Xingyue $\mathrm{Li}^{\mathrm{a}}$, Lixia Qin ${ }^{\mathrm{a}}$, Miao Zhang ${ }^{\mathrm{a}}$, \\ Guangjian Liu ${ }^{\mathrm{a}}$, Xiaodong Liu ${ }^{\mathrm{a}}$
}

\begin{abstract}
Meningeal carcinoma has a variety of clinical manifestations and a high mortality and disability rate. Among them, cranial hypertension is one of the important causes of death, which is easy to be missed or misdiagnosed. The purpose of this case report is to describe the clinical features of diffuse leptomeningeal glioneuronal tumor (DNGNT) with the first symptom of headache accompanied by decreased vision misdiagnosed as venous sinus thrombosis. The patient initially suffered from headache and decreased vision, and rapidly developed blindness. According to the clinical manifestations and cerebrospinal fluid (CSF) examination results, the patient was initially misdiagnosed as venous sinus thrombosis, the intracranial pressure was relieved by Ommaya capsule, and the cerebrovascular condition was observed by magnetic resonance venogram (MRV) and digital subtraction angiography (DSA). The degree of meningeal enhancement was observed by enhanced magnetic resonance imaging (MRI), and meningeal cancer cells were found by CSF cytology. The patient was excluded from viral meningitis, tuberculous meningitis, cryptococcal meningitis, optic neuromyelitis, and central nervous system Behcet's disease by routine examination. Ommaya capsule implantation relieved the intracranial hypertension, and vision was restored after treatment. No venous sinus thrombosis was found in MRV and DSA; brain-enhanced MRI revealed extensive linear enhancement of the meninges, and CSF cytology revealed grotesque, actively dividing tumor cells; she was diagnosed as DLGNT. Meningeal carcinoma cannot be excluded in adolescents without tumor history. When patient has unexplained intracranial hypertension, it is necessary to consider DLGNT; early brain-enhanced MRI and CSF cytology are helpful for early diagnosis. For cranial hypertension, timely placement of Ommaya capsule can improve patient quality of life and save vision in time; molecular targeted therapy as early as possible can prolong survival.
\end{abstract}

Manuscript submitted May 30, 2019, accepted June 19, 2019

aDepartment of Neurology, Taihe Hospital Affiliated to Hubei University of Medicine, Shiyan, Hubei 442000, China

${ }^{b}$ Corresponding Author: Yi Bao, Department of Neurology, Taihe Hospital Affiliated to Hubei University of Medicine, Shiyan, Hubei 442000, China. Email: karlbaoyi@163.com

doi: https://doi.org/10.14740/jnr539
Keywords: Diffuse leptomeningeal glioneuronal tumor; Venous sinus thrombosis; Ommaya sac; Cytology; Digital subtraction angiography

\section{Introduction}

In 1912, Beer Man first named a tumor of the central nervous system characterized by headache and vomiting as meningeal cancer. It is a metastatic tumor, which mainly diffuses into the pia mater and subarachnoid space. The primary lesions are often from lung cancer, breast cancer and hematological system tumors. As the disease progresses, the lesion often involves the cerebral cortex, cranial nerves, spinal cord, and spinal nerve roots. The mortality and disability of it are extremely high, and intracranial hypertension is one of the important causes of death $[1,2]$.

Due to the low incidence and atypical clinical symptoms, the diagnosis requires the use of cerebrospinal fluid (CSF) cytology to find cancer cells, but the positive rate is extremely low. It is often misdiagnosed as infectious diseases (such as cryptococcal meningitis, viral meningitis, tuberculous meningitis), non-infectious diseases (autoimmune encephalitis, central system Behcet's disease, venous sinus thrombosis) and other diseases before the cancer cells are found. If the manifestation is visual impairment, it can also be misdiagnosed as optic neuromyelitis, Vogt-Koyanagi-Harada syndrome, multiple sclerosis, and so on [3, 4].

Cerebral venous sinus thrombosis (CVST) is a rare and serious neurological disease, mainly due to obstruction of cerebral venous, venous sinus reflux, and its incidence accounts for $0.5 \%$ of stroke. CVST tends to occur in young and middleaged people, with acute/subacute and chronic onset. Obstruction of cerebral venous reflux leads to increased intracranial pressure. Most intracranial pressure is over $300 \mathrm{~mm} \mathrm{H}_{2} \mathrm{O}(1$ $\mathrm{mm} \mathrm{H}_{2} \mathrm{O}=0.0098 \mathrm{kPa}$ ), which is manifested as headache, vomiting, papilledema, accompanied or not accompanied by consciousness disorder, neurological dysfunction. Headache of different degrees is often the first symptom, which is characterized by severe illness and high disability mortality $[5,6]$. With the development of neuroimaging technology and the maturation of treatment, the disease is gradually recognized. 
The skull magnetic resonance imaging (MRI) and magnetic resonance venogram (MRV) can visualize the intracranial venous sinus cavity and large veins, which are the preferred imaging evaluation methods for the diagnosis of CVST and the detection of efficacy. Digital subtraction angiography (DSA) is regarded as the "gold standard" for the assessment of intracranial arteriovenous vascular disease. Although it has good sensitivity and specificity, it is an invasive examination. The clinical manifestations of CVST are atypical and prone to misdiagnosis and missed diagnosis, and the diagnosis and treatment of CVST are still a difficult problem for clinicians [7, 8].

We described a case of adolescent girl with headache, vision loss and rapid blindness. The lumbar puncture examination showed that the CSF pressure was extremely high, and the CSF routine test and biochemical results were not specific. The test results of multiple antibodies, smears and pathogens were negative. After the diagnostic antiviral, anti-Cryptococcus, anti-tuberculosis therapy and immunotherapy, the symptoms had not been improved significantly and the venous sinus thrombosis was suspected. But the results were negative by MRV and DSA. Finally, diffuse leptomeningeal glioneuronal tumor (DLGNT) was diagnosed by enhanced MRI and CSF cytology. The specific misdiagnosis is now shared as follows. The differential diagnosis of this patient with optic neuromyelitis [9], tuberculous meningoencephalitis, cryptococcal meningitis, and related CSF dynamic changes and spinal cord metastasis will be discussed in detail in other articles.

\section{Case Report}

The patient was a 16-year-old adolescent girl, a high school student. The first symptoms were headache, nausea and vomiting, accompanied by vision loss. After the diagnosis and treatment in the local clinic, there was no improvement, and the vomiting was aggravated. It was spray-like and the visual acuity decreased significantly. Therefore, the patient came to the neurology department of our hospital for treatment. She was previously healthy without any special medical history or surgical trauma history. Physical examination showed that the vital signs were stable, and no positive signs were found in the cardiopulmonary examination. Neurological examination showed lethargy, right eyelid dropping, unequal bilateral pupils (right/left $=5 / 4$ ), insensitiveness to light reflection, meningeal stimulation positive, with no other positive signs of neurological examination.

\section{Routine examination}

After admission, the patient was examined by head computed tomography $(\mathrm{CT})$, chest $\mathrm{CT}$, electrocardiogram, blood analysis, and was tested for erythrocyte sedimentation rate (ESR), $\mathrm{C}$-reactive protein (CRP), tumor markers, autoimmune antibody spectrum, thyroid function, tuberculosis antibody, TBSPOT, virus antibody, liver and kidney function, electrolyte, coagulation function and received other examinations. Blood routine, ESR, CRP, tuberculosis antibody, TB-SPOT, viral antibody, liver and kidney function, electrolyte, coagulation function tests and other tests showed no obvious abnormalities. Tumor markers, autoimmune antibody profiles, and thyroid function were all within the normal range. No obvious lesions were seen in the head CT.

\section{CSF examination}

After informed consent of the patient and her family members was obtained, local anesthesia with lidocaine was used, and the needle was inserted vertically at the L3-4. In order to avoid cerebral hernia, we stopped pressure measurement. Under the condition of using needle core to control the flow rate, CSF was collected and sent for routine, biochemical, acid-fast staining, ink staining, tested for antibodies related to optic neuromyelitis, oligoclonal band, autoimmune encephalitis, and it was sent to Huada Gene (Beijing, China) to check the pathogenic gene fragments of viruses, bacteria, fungi, tuberculosis bacilli and parasites.

The cerebrospinal fluid pressure was over $400 \mathrm{~mm} \mathrm{H}_{2} \mathrm{O}$. CSF routine test showed light yellow, mild turbid CSF, no clot, Pan's test positive, total cell number of 20, white blood cells (WBCs) of 13. CSF smear showed new cryptococcal polysaccharide antigen negative, with no acid-fast bacilli. Ink staining was negative. CSF biochemistry showed Pro $4.97 \mathrm{~g} / \mathrm{L}, \mathrm{Cl} 105.2$ $\mathrm{mmol} / \mathrm{L}$, Glu $4.98 \mathrm{mmol} / \mathrm{L}$, LDH 34, hsCRP $0.37 \mathrm{mg} / \mathrm{L}$, ADA $0.41 \mathrm{U} / \mathrm{L}$. Neuromyelitis optica spectrum disorder (NMOSD)related antibody test showed that AQP-4, NMO-IgG, MOG were negative. Autoimmune encephalitis-related antibody test showed that NMDA-R-Ab, CASPR2-Ab, AMPA1-R-Ab, AMPA2-R-Ab, LGI1-Ab, GABA-R-Ab, GAD65-Ab were negative. Oligoclonal band was negative. The results of the inspection of Huada Gene Company showed that no pathogenic gene fragments such as viruses, bacteria, fungi, mycobacterium tuberculosis, and parasites were found.

The patient's intracranial pressure was extremely high, nausea and vomiting were severe, and visual acuity was significantly reduced. After the ophthalmic consultation, the optic disc edema was considered, and the reason was intracranial hypertension. To save vision and avoid optic nerve atrophy, neurosurgical emergency Ommaya capsular implantation was performed to drain CSF and release intracranial pressure.

In response to the ultra-high intracranial pressure and increased CSF protein, diagnostic antiviral, anti-tuberculosis, anti-Cryptococcus therapies and immunotherapy (intravenous use of gamma globulin combined with methylprednisolone) had been successively performed, and the condition was not improved. It was necessary to consider venous sinus thrombosis, and to give low molecular weight heparin subcutaneous injection to prevent thrombosis, and to actively find the cause.

\section{MRI and MRV examination}

Since the patient had extremely high intracranial pressure, CSF was not specific, and no pathogens were found. Brain MRI and MRV were used to exclude venous sinus thrombosis. Plain scan and enhanced examination were performed using 

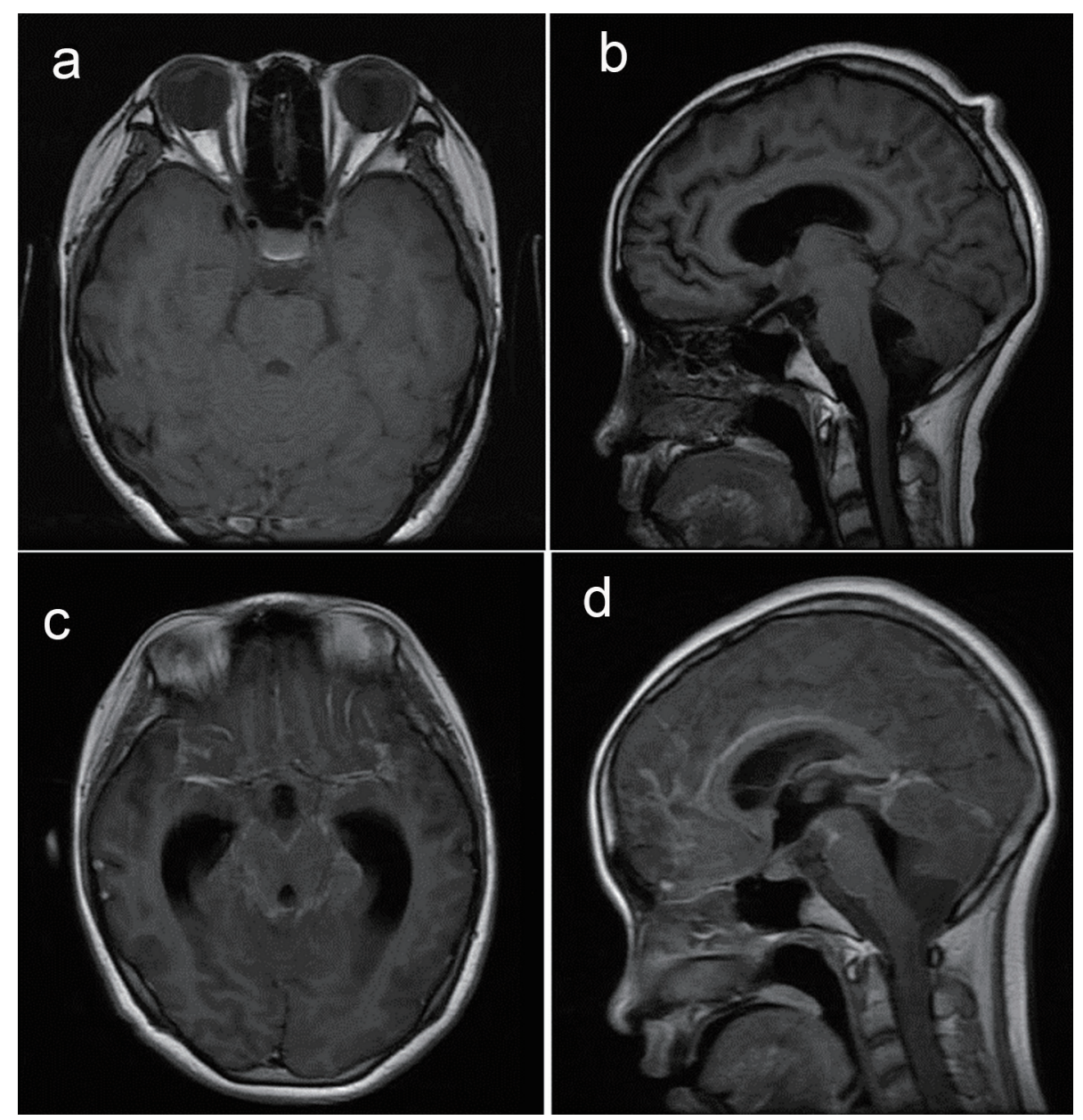

Figure 1. Brain magnetic resonance imaging and enhancement results. Contrast can be seen in meningeal enhancement.

symphoy-p3.0T MRI instrument of Siemens Company (Erlangen, Germany): axial, sagittal and coronal images were collected from the brain using Gd-DTPA according to the body weight of $\times 0.1 \mathrm{mmol} / \mathrm{kg}$.

Plain brain scan (Fig. 1a, b) showed abnormal signals in bilateral hippocampus and right thalamus. There was a high possibility of virus infection and slightly enlarged supratentorial ventricle. After enhancement, the right thalamic lesion and meninges showed lamellar enhancement, with meninges in the basal cisterna of the brain as the focus, ventricular system dilation, and midline structure was basically centered (Fig. 1c, d). No abnormalities were found in the brain MRV scan (Fig. 2a, b).

\section{Cerebral angiography}

In order to further understand the situation of venous sinus thrombosis, the cerebral angiography was performed in the supine position after obtaining informed consent of the patient's family. After routine disinfection and spreading, local anesthesia and systemic heparinization were performed. The modified femorization method was used to perform the right femoral artery puncture, $5 \mathrm{~F}$ arterial sheath, and intubation. The aortic arch, bilateral subclavian, total neck, intracranial and vertebral angiography were performed to determine the location and extent of cerebral venous sinus stenosis (CVSS) and to observe the presence or absence of dilatation and arteriovenous circulation time. After the operation, the sheath was removed with a pressure-wrapped puncture site. The patient was returned to the ward, and the patient's right lower limb was braked for $24 \mathrm{~h}$.

Cerebral angiography showed bilateral common carotid artery, subclavian artery, intracranial segment of internal carotid artery, bilateral external carotid artery, middle cerebral artery, anterior cerebral artery, bilateral vertebral artery intracranial segment, basilar artery, good imaging of bilateral posterior cerebral artery, and no obvious stenosis. The arteriovenous phase was well shown (Fig. 2c, d).

\section{CSF cytology}

In order to find tumor cells, fresh CSF was taken through Ommaya capsule, and $500 \mu \mathrm{L}$ of pellet was taken after centrifugation at $4{ }^{\circ} \mathrm{C}$. The cells of CSF were enriched by slide centrifugation using FMU-6 cell slides. After dried, it was dyed by Wright's staining $(\times 400)$. After $10 \mathrm{~min}$, we rinsed off the back- 

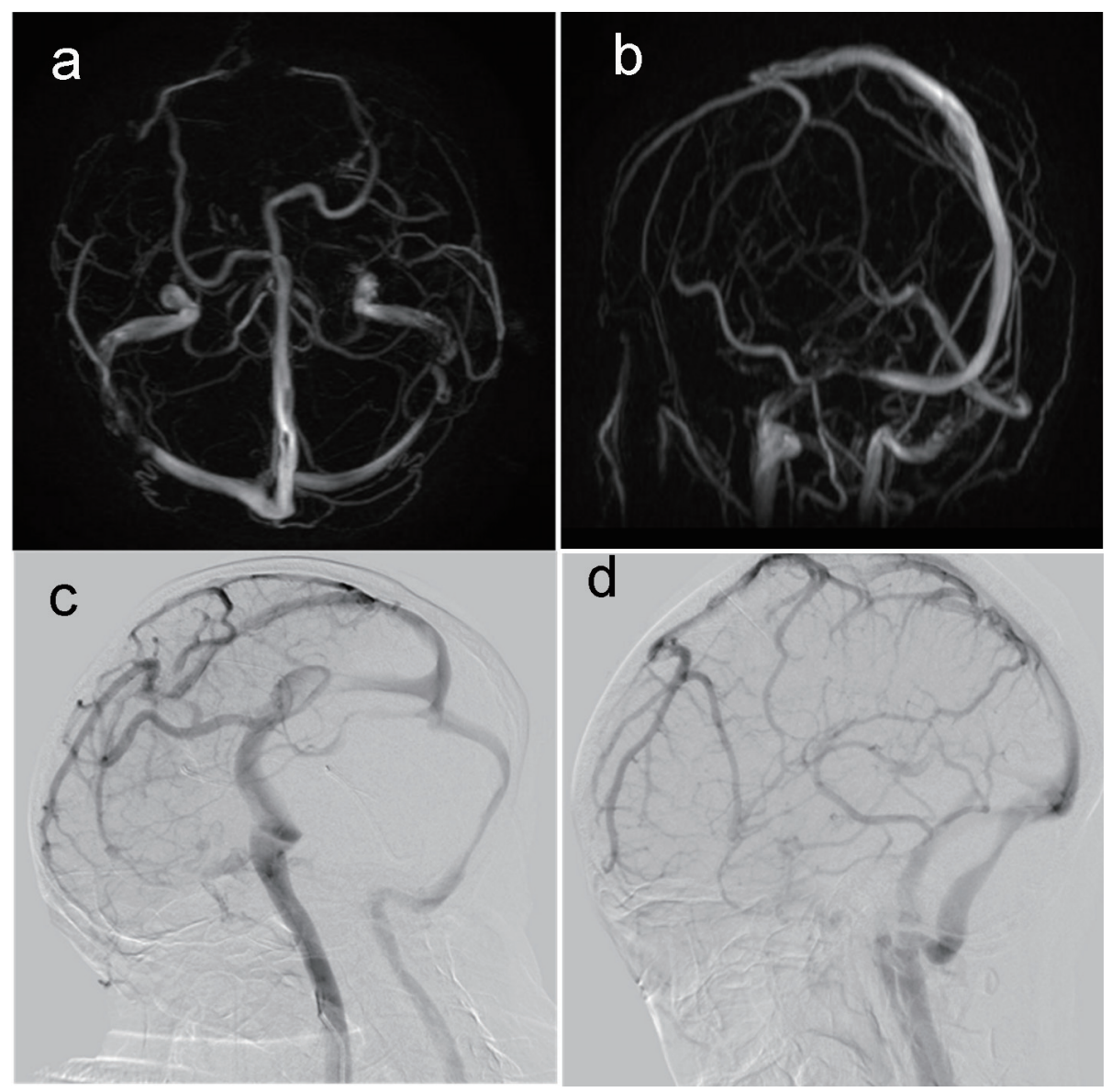

Figure 2. (a, b) MRV showed no venous sinus thrombosis; (c, d) DSA showed no venous sinus thrombosis.

ground dye with running water, first looked for cancer cells under low magnification, and then used the oil microscope to observe the cell morphology.

After repeated cytological examination of cancer cells, through Wright's staining $(\times 400)$, it could be seen that the tumor cells were malformed, irregularly arranged, and of different sizes; the cell membrane had protrusions and the shape was obvious. There were vacuoles and pigment particles in the cytoplasm; the nucleus accounted for the majority of the cell volume, with active mitosis and obvious lobes; and the nucleolus was obvious (Fig. 3).

\section{Progress and outcome}

According to CSF cytology and enhanced MRI, the patient
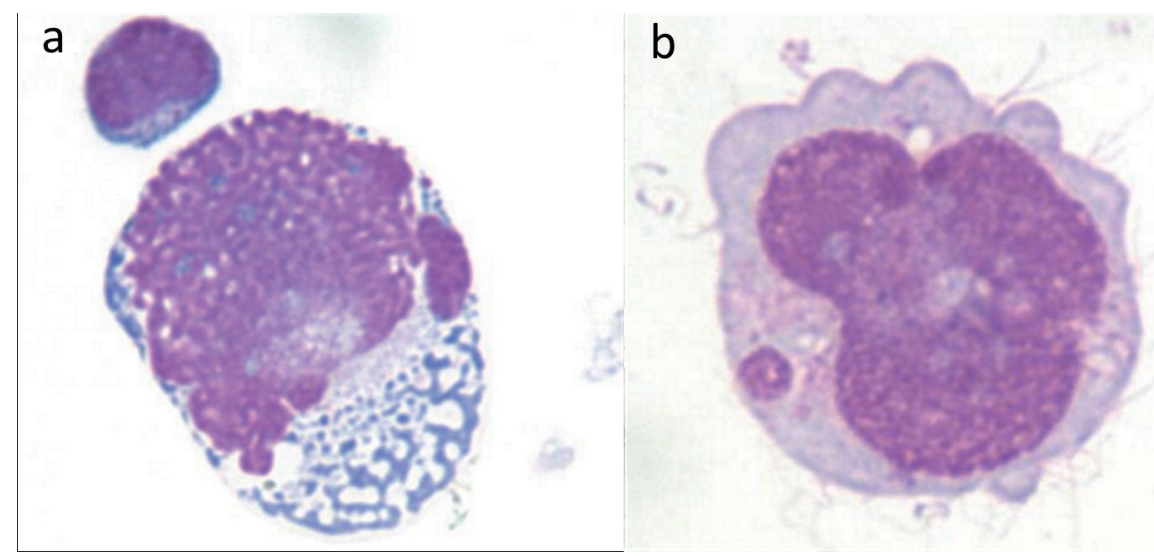

Figure 3. CSF cytology (Wright's staining $\times$ 400) found cancer cells with abnormal shape, active mitosis, and nucleotosis. 
was diagnosed as DLGNT. During hospitalization, the patient gradually developed numbness in both lower extremities and urinary retention. The family members refused further brain biopsy and PET-CT examination after learning that the prognosis was poor, and asked to return to the local hospital for treatment. After they left the hospital, we learned through multiple phone interviews that the patient's condition gradually progressed, and the lower limbs could not walk and she was sensory deprived.

\section{Discussion}

Common symptoms of DLGNT are headache, nausea, vomiting, and progressive exacerbations. Usually, the body can be found to have positive meningeal irritation, papilledema, positive pyramidal tract, decreased muscle strength, visual hearing loss, and dyskinesia, increased CSF pressure, increased cell number and protein, reduced sugar and chlorine, and the tumor cells are seen in cytological examination. Currently, the diagnostic criteria of DLGNT are as follows: 1) A history of tumor; 2) A new meningeal irritation; 3) CSF cytology to detect cancer cells; and 4) Typical MRI performance, where 1), 2), plus one of 3) or 4), can be diagnosed. Enhanced MRI can find local or diffuse enhancement of the meninges, ependymal and perivascular space, which has an important early diagnostic value. CSF cytology is the gold standard for diagnosis for it can find cancer cells $[10,11]$. Due to the diverse clinical manifestations and non-specificity, meningeal cancer is difficult to be diagnosed, but easy to be misdiagnosed and confused with sinus thrombosis.

The clinical manifestations of intracranial venous sinus thrombosis are quite different, often manifested as nausea, vomiting, transient blurred vision, disturbance of consciousness, and so on, and can also be manifested as a series of focal neurological symptoms, including seizures, optic disc edema, and subarachnoid hemorrhage $[12,13]$. The cause was divided into infectious and non-infectious factors. Infectious factors are mostly caused by cavernous sinus, transverse sinus, and sigmoid sinus thrombosis, often secondary to infections of the face, eyes, ears, nose, pharynx, and paranasal sinuses. The main causes of non-infectious CVST formation are heart disease, puerperium, blood diseases and long-term oral contraceptives. According to the onset time, it can be divided into acute, subacute and chronic processes, with high disability rate and mortality $[14,15]$. This case was a young female patient, consistent with the common age of venous sinus thrombosis. Her first symptoms were headache, vomiting, decreased vision, and high intracranial pressure, similar to the clinical manifestations of venous sinus thrombosis.

The patient was excluded from viral meningitis, tuberculous meningitis, cryptococcal meningitis, optic neuromyelitis, and central nervous system Behcet's disease. After the treatment with mannitol and glycerol fructose dehydration, the patient's symptoms did not improve. After the implantation of the Ommaya capsule, the patient had a large amount of CSF drainage per day. As the drug treatment progressed, the daily drainage volume was not significantly reduced. According to the patient's condition, venous sinus thrombosis was considered, but the point of no support was associated with no infectious and noninfectious factors. In order to diagnose the venous sinus thrombosis, it was proposed to use MRV for examination. The current skull MRI and MRV can visualize the intracranial venous sinus cavity and large veins as the preferred imaging assessment method for the diagnosis of CVST. However, MRV shows sinus blood flow imaging, which is obviously affected by blood flow velocity and artifacts. It is inaccurate for slow venous sinus and venules. Loss of signal sometimes does not mean blood flow stagnation or thrombosis [16-18]. A thrombus was not found in the patient in the MRV examination. Considering the influence of blood flow velocity and artifacts on the MRV examination, the venous sinus thrombosis could not be completely ruled out, so a more accurate examination method should be taken for further investigation. At this stage, DSA is the gold standard for the diagnosis of CVST, which can clearly show the condition of thrombus expansion, the length of development, the degree of collateral circulation, blood stasis, affected lesion filling defect and sinus wall irregularity. DSA has a better sensitivity and specificity. After informed consent was obtained, DSA examination was performed, but the result was negative.

The patient's intracranial hypertension mainly manifests as an intense headache, jet vomiting, papilledema, loss of vision, and decreased consciousness. If it cannot be relieved for a long time, it is easy to cause optic atrophy, permanent blindness, and a risk of cerebral palsy. The patient was scheduled to be diagnosed with venous sinus thrombosis and needed the urgent treatment. At present, the treatment of venous sinus thrombosis is mainly divided into drug treatment and endovascular treatment. The effects of drug treatment include antiinflammation, dehydration, thrombolysis and anticoagulation. In the absence of contraindications to anticoagulant drugs, anticoagulant therapy is preferred. Heparin is currently the firstline treatment for CVST and has good efficacy and safety. The main drug for intravenous thrombolytic therapy is urokinase. Endovascular treatment includes sinus contact thrombolysis, mechanical thrombectomy, balloon scutellum, and solitaire stent thrombectomy. In addition, for patients with ultra-high intracranial pressure, decompressive craniectomy is used to prevent cerebral palsy $[6,19-20]$. No thrombus was found in the patient, but low molecular weight heparin anticoagulation was still used, and timely consultation with ophthalmology and neurosurgery was performed. The Ommaya capsule implantation was operated, combined with dehydration, nutritional nerves, scavenging oxygen free radicals, improving circulation, hyperbaric oxygen, and so on; after a timely treatment, the patient's vision was restored to normal. Heparin also helped the patient's vision recovery, and reduced the risk of deep vein thrombosis of the lower extremity caused by prolonged bed rest. This improved the quality of life and prolonged her survival, which is the success of this case.

Because no thrombus was found in MRV and DSA examinations, the patient's CSF gradually showed decreased transparency, darkened color, increased protein, and clot formation, which was inconsistent with CSF changes caused by sinus thrombosis. In order to further identify the cause, brainenhanced MRI was checked and extensive linear enhancement of meninges and spinal cord membranes was found. CSF cy- 
tology found grotesque, actively dividing tumor cells, so the patient was diagnosed as DLGNT. However, the patient was an adolescent girl with no history of oncology, which was inconsistent with DLGNT with a history of tumor and metastatic infiltration of the meninges. Acute onset was rare in DLGNT. CSF pressure was extremely high as well as the protein, and sugar and chloride were not significantly decreased, which was different from the CSF of DLGNT. This is a special feature of this case.

Intracranial hypertension is the main cause of death in DLGNT, and its mechanism is that CSF reflux is blocked due to infiltration of cancer cells, resulting in hydrocephalus and damage of cranial nerve or spinal nerve root. When mannitol dehydration was ineffective in reducing cranial pressure, Ommaya sac implantation was performed in a timely manner to drain the excess CSF every day, which significantly improved the patient's symptoms and improved the patient's quality of life.

The shortcomings of this study are listed as follows. As it is a single-sample clinical case, the success of reducing intracranial pressure, visual acuity treatment and prevention of venous thrombosis in lower limbs still needs the support of a large number of clinical samples. The final diagnosis requires a brain biopsy for accurate typing. After the diagnosis of DLGNT, because the patient gave up treatment, there was a lack of followup relevant treatment data. The differential diagnosis of this case and other diseases will be elaborated in our subsequent articles.

\section{Conclusions}

DLGNT is rare, there is no effective cure, and the mortality rate is high. Early diagnosis can improve the quality of life and prolong survival [21-24]. However, in the current situation, clinical symptoms are not typical, and the first doctor lacks relevant clinical experience. The current positive rate of CSF cytology is low, and the treatment regimen is ineffective. Accordingly, through this case report, we intend to increase the information related to DLGNT, describe the diagnosis and misdiagnosis, helping clinicians learn and understand the disease. When patients have an unexplained intracranial hypertension, if the treatment is not effective, regardless of whether there is a history of cancer, it is recommended to take brainenhanced MRI and CSF cytology as early as possible to understand whether there is the possibility of DLGNT. In terms of treatment, timely placement of Ommaya capsules can help improve patient symptoms, and molecular targeted therapy offers new hope for prolonging the life of patients [25-27].

\section{Acknowledgments}

We are grateful to the medical staff who have treated this patient carefully.

\section{Financial Disclosure}

None.

\section{Conflict of Interest}

None.

\section{Informed Consent}

Informed consent has been obtained.

\section{Author Contributions}

$\mathrm{YB}, \mathrm{YH}$ and YD contributed to data collection; YB, YH and $\mathrm{XL}$ contributed to data analysis; YB, YH, YD, LQ and $\mathrm{MZ}$ contributed to manuscript preparation; YH, XL, LQ, MZ, GL, $\mathrm{XL}$, and YB reviewed manuscript.

\section{References}

1. Gauthier H, Guilhaume MN, Bidard FC, Pierga JY, Girre V, Cottu PH, Laurence V, et al. Survival of breast cancer patients with meningeal carcinomatosis. Ann Oncol. 2010;21(11):2183-2187.

2. de Azevedo CR, Cruz MR, Chinen LT, Peres SV, Peterlevitz MA, de Azevedo Pereira AE, Fanelli MF, et al. Meningeal carcinomatosis in breast cancer: prognostic factors and outcome. J Neurooncol. 2011;104(2):565-572.

3. Subira D, Simo M, Illan J, Serrano C, Castanon S, Gonzalo R, Granizo JJ, et al. Diagnostic and prognostic significance of flow cytometry immunophenotyping in patients with leptomeningeal carcinomatosis. Clin Exp Metastasis. 2015;32(4):383-391.

4. Palma JA, Fernandez-Torron R, Esteve-Belloch P, Fontes-Villalba A, Hernandez A, Fernandez-Hidalgo O, Gallego Perez-Larraya J, et al. Leptomeningeal carcinomatosis: prognostic value of clinical, cerebrospinal fluid, and neuroimaging features. Clin Neurol Neurosurg. 2013;115(1):19-25.

5. Borhani Haghighi A, Edgell RC, Cruz-Flores S, Feen E, Piriyawat P, Vora N, Callison RC, et al. Mortality of cerebral venous-sinus thrombosis in a large national sample. Stroke. 2012;43(1):262-264.

6. Misra UK, Kalita J, Chandra S, Kumar B, Bansal V. Low molecular weight heparin versus unfractionated heparin in cerebral venous sinus thrombosis: a randomized controlled trial. Eur J Neurol. 2012;19(7):1030-1036.

7. Roh D, Carroll J, Melmed K, Elkind MSV, Eisenberger A, Francis R, Park S, et al. Endovascular Treatment of Cerebral Venous Sinus Thrombosis and Insights into Intracranial Coagulopathy. J Stroke Cerebrovasc Dis. 2019;28(4):e7-e9.

8. Capecchi M, Abbattista M, Martinelli I. Cerebral venous sinus thrombosis. J Thromb Haemost. 2018;16(10):19181931.

9. Xiao J, Gao L, Zhang M, et al. Clinical features of diffuse leptomeningeal glioneuronal tumor with rapid blindness misdiagnosed as NMOSD and literature review. SN Com- 
prehensive Clinical Medicine. 2019;1(6):434-441.

10. Engelhard HH, Villano JL, Porter KR, Stewart AK, Barua M, Barker FG, Newton HB. Clinical presentation, histology, and treatment in 430 patients with primary tumors of the spinal cord, spinal meninges, or cauda equina. J Neurosurg Spine. 2010;13(1):67-77.

11. Chamberlain MC. Leptomeningeal metastasis. Curr Opin Oncol. 2010;22(6):627-635.

12. Sebire G, Tabarki B, Saunders DE, Leroy I, Liesner R, Saint-Martin C, Husson B, et al. Cerebral venous sinus thrombosis in children: risk factors, presentation, diagnosis and outcome. Brain. 2005;128(Pt 3):477-489.

13. Gosk-Bierska I, Wysokinski W, Brown RD, Jr., Karnicki K, Grill D, Wiste H, Wysokinska E, et al. Cerebral venous sinus thrombosis: Incidence of venous thrombosis recurrence and survival. Neurology. 2006;67(5):814-819.

14. Wang JW, Li JP, Song YL, Tan K, Wang Y, Li T, Guo P, et al. Clinical characteristics of cerebral venous sinus thrombosis. Neurosciences (Riyadh). 2015;20(3):292-295.

15. Devasagayam S, Wyatt B, Leyden J, Kleinig T. Cerebral Venous Sinus Thrombosis Incidence Is Higher Than Previously Thought: A Retrospective Population-Based Study. Stroke. 2016;47(9):2180-2182.

16. Connor SE, Jarosz JM. Magnetic resonance imaging of cerebral venous sinus thrombosis. Clin Radiol. 2002;57(6):449-461.

17. Qu H, Yang M. Early imaging characteristics of 62 cases of cerebral venous sinus thrombosis. Exp Ther Med. 2013;5(1):233-236.

18. Altinkaya N, Demir S, Alkan O, Tan M. Diagnostic value of T $2 *$-weighted gradient-echo MRI for segmental evaluation in cerebral venous sinus thrombosis. Clin Imaging. 2015;39(1):15-19.

19. Ilyas A, Chen CJ, Raper DM, Ding D, Buell T, Mastorakos P, Liu KC. Endovascular mechanical thrombectomy for cerebral venous sinus thrombosis: a systematic review. J Neurointerv Surg. 2017;9(11):1086-1092.
20. Ma J, Shui S, Han X, Guo D, Li TF, Yan L. Mechanical thrombectomy with Solitaire AB stents for the treatment of intracranial venous sinus thrombosis. Acta Radiol. 2016;57(12):1524-1530.

21. Kak M, Nanda R, Ramsdale EE, Lukas RV. Treatment of leptomeningeal carcinomatosis: current challenges and future opportunities. J Clin Neurosci. 2015;22(4):632637.

22. Meng Z, Zhang Q, Hong K, Han W, Zhao Z, Liu Y, He J, et al. Clinical outcome and prognostic analysis of meningeal carcinomatosis treated by intrathecal chemotherapy. Expert Rev Pharmacoecon Outcomes Res. 2018;18(4):455460.

23. Montes de Oca Delgado M, Cacho Diaz B, Santos Zambrano J, Guerrero Juarez V, Lopez Martinez MS, Castro Martinez E, Avendano Mendez-Padilla J, et al. The Comparative Treatment of Intraventricular Chemotherapy by Ommaya Reservoir vs. Lumbar Puncture in Patients With Leptomeningeal Carcinomatosis. Front Oncol. 2018;8:509.

24. Corradini S, Hadi I, Hankel V, Ertl L, Ganswindt U, Belka C, Niyazi M. Radiotherapy of spinal cord gliomas : A retrospective mono-institutional analysis. Strahlenther Onkol. 2016;192(3):139-145.

25. Lee DW, Lee KH, Kim JW, Keam B. Molecular Targeted Therapies for the Treatment of Leptomeningeal Carcinomatosis: Current Evidence and Future Directions. Int $\mathbf{J}$ Mol Sci. 2016;17(7):1074.

26. Jiao XD, Ding C, Zang YS, Yu G. Rapid symptomatic relief of HER2-positive gastric cancer leptomeningeal carcinomatosis with lapatinib, trastuzumab and capecitabine: a case report. BMC Cancer. 2018;18(1):206.

27. Yu HA, Tian SK, Drilon AE, Borsu L, Riely GJ, Arcila ME, Ladanyi M. Acquired resistance of EGFR-mutant lung cancer to a T790M-specific EGFR inhibitor: Emergence of a third mutation (C797S) in the EGFR Tyrosine kinase domain. JAMA Oncol. 2015;1(7):982-984. 